\title{
Análisis estadístico implicativo de los conocimientos previos sobre propiedades de operaciones con números reales de ingresantes a carreras de ingeniería de FACENA
}

Prof. María E. Mendoza; Esp. Liliana N. Caputo; Mgter Eduardo A. Porcel (*)

\section{RESUMEN}

Este trabajo tiene por objetivo presentar un método de Estadística multivariada llamado Análisis Estadístico Implicativo (ASI) que permite determinar los conocimientos de los estudiantes (materializados en sus respuestas a distintos ítems de evaluación) así como las relaciones conceptuales que entre ellos establecen los sujetos evaluados. ASI, a diferencia de los métodos clásicos de asociación de variables, permite detectar relaciones del tipo "si p, entonces, casi siempre q", a las cuales se las denomina cuasi-implicaciones o reglas. Estas relaciones se presentan mediante un "grafo implicativo" que permite la mejor interpretación de las relaciones mencionadas.

Para lograr el objetivo propuesto en este artículo, se utilizó ASI para analizar un ítem de evaluación de una prueba de diagnóstico suministrada a ingresantes a Ingeniería Eléctrica, Ingeniería en Agrimensura e Ingeniería en Electrónica al inicio del año lectivo 2017 y se realizó una comparación entre las relaciones detectadas según los alumnos pertenezcan a una u otra carrera.

A modo general, se observaron saberes y relaciones conceptuales establecidos por los alumnos que dan cuenta de su escasa evolución del pensamiento aritmético al algebraico, independientemente de la carrera a la que pertenecen.

Palabras clave: Cuasi-implicaciones. Grafo implicativo. Ingresantes a ingenierías. Operaciones con Números Reales

\section{INTRODUCCIÓN}

Al implementar una evaluación, los docentes esperan obtener información respecto a si los sujetos evaluados han construido ciertos saberes. Para ello realizan un análisis previo para determinar qué conceptos o propiedades se deben poner en juego en cada ítem de evaluación. Sin embargo, generalmente, las respuestas de los alumnos no coinciden con las expectativas del evaluador $y$, por lo general éste no logra

(*) Facultad de Ciencias Exactas y Naturales y Agrimensura (FACENA) - (UNNE)

\{mat.mendoza2812,proflcaputo, esteporcel\}@gmail.com 
comprender cuáles son las relaciones conceptuales que constituyen la red cognitiva del sujeto evaluado.

Para hallar estas relaciones, a fines del siglo pasado, el grupo de investigación de Régis Gras (perteneciente a la Universidad de Nantes, Francia) creó un método de análisis estadístico multivariada llamado Analyse Statisque Implicative (ASI) el cual, traducido al castellano, se denomina Análisis Estadístico Implicativo. Este método, a diferencia de las técnicas clásicas de asociación de variables, establece entre las mismas (los ítems de evaluación) relaciones no simétricas denominadas cuasi-implicaciones o reglas.

En este artículo, se presenta este método y se ejemplifica su uso mediante el análisis de un ítem correspondiente a una evaluación de diagnóstico referida a números reales, suministrada a ingresantes a

Ingeniería Eléctrica, Ingeniería en Agrimensura e Ingeniería en Electrónica al inicio del ciclo lectivo 2017.

\section{DESARROLLO}

\subsection{Metodología}

ASI, partiendo de la hipótesis: "Si un ejercicio es más complejo que otro, entonces todo alumno que resuelve el primero debería resolver también el segundo" [1] establece relaciones del tipo "si I, entonces, casi siempre $I_{k}$ " $\left(I_{j} \Rightarrow I_{k}\right)$ a las que se llaman cuasi - implicaciones o reglas.

Para su aplicación ASI requiere, en principio, de los conjuntos finitos: el con- junto de variables dicotómicas $V=\left\{\mathrm{I}_{1}, \ldots, \mathrm{I}_{\mathrm{n}}\right\}$ (formado por los ítems de evaluación) y el conjunto $E$, de los sujetos evaluados. De esta manera, se tiene que si $\mathrm{x} \in E$ resolvió correctamente el ítem $\mathrm{k}(1 \leq \mathrm{k} \leq \mathrm{n}) \mathrm{I}_{\mathrm{k}}(\mathrm{x})=1$; en caso contrario ( si contestó mal o no contestó) $I_{k}(x)=0$.

Entonces, dados $I_{j} I_{k} \in V$, en la lógica clásica $I_{j} \Rightarrow I_{k}$ es verdadera si, siendo $I(x)=1, I_{k}(x)=1, \forall \mathrm{x} \in E$. Es decir, $A, A_{k}$ siendo $A_{j}=\left\{x \in E / I_{j}(x)=1\right\}$ y $A_{k}=\left\{x \in E / I_{k}(x)=1\right\}$. Sin embargo, en la realidad no siempre sucede que sea suficiente que $I_{j}(x)=1$ para que también lo sea $I_{k}(x)$ por lo cual ASI para establecer si existe o no relación entre $I_{j}$ e $I_{k}$ minimiza el peso de los contraejemplos esto es, el peso de los casos en que $I_{j}(x)=1$ e $I_{k}$ $(x)=0$. La existencia de dichos contraejemplos, permite afirmar que $A_{j}-A_{k} \neq \varnothing$.

Entonces, si $X$ e $Y$ son dos subconjuntos de $E$ tales que $\operatorname{car}(X)=\operatorname{car}(A) \mathrm{y}$ $\operatorname{car}(Y)=\operatorname{car}\left(A_{k}\right)$, Gras y Kunt [2] establecen que $A_{j} \Rightarrow A_{k}$ sea admisible a nivel de confianza $1-\alpha$ equivale a que $\operatorname{Pr}\left[\operatorname{car}(X-Y) \leq \operatorname{car}\left(A_{j}-\right.\right.$ $\left.\left.A_{k}\right)\right] \leq \alpha$. Estas probabilidades se calculan usando la distribución Hipergeométrica, Binomial o de Poisson, según sea el tipo de población y el tamaño de muestra [3]. Sin embargo, bajo ciertas condiciones las primeras distribuciones tienden a la ley de Poisson de parámetro

$$
\lambda=\frac{\operatorname{car}\left(A_{j}\right) \cdot \operatorname{car}\left(E-A_{k}\right)}{\operatorname{car}(E)}[4] .
$$

Por otra parte, se define un índice que estima la diferencia entre $\operatorname{car}\left(A_{j}-A_{j}\right)$ y el valor que habría tomado si $I_{j}$ e $I_{k}$ fueran independientes. Dicha diferencia se apro- 
xima a la normal $(0,1)$ por lo cual, se define la intensidad de la implicación $I_{j} \Rightarrow I_{k}$ utilizando la función de densidad de dicha distribución, a la que se denota $\phi\left(I_{j} I_{k}\right)$. De esta manera, se dice que $I_{j} \Rightarrow I_{k}$ es admisible a nivel de confianza $1-\alpha$, si $\phi\left(I_{j} I_{k}\right) \geq 1-\alpha$.

Grass y Kuntz [2] observaron que si el número de sujetos evaluados (\#(E)) es muy grande, el método antes descripto pierde efectividad, pues el aumento del número de contraejemplos vulnera la intensidad de las cuasi-implicaciones (a las que los autores denominaron clásicas). Por tal motivo, apelando al concepto de entropía de Shannon, definieron un índice que da cuentas de la admisibilidad de la implicación y de su contrarrecíproca, a partir de un número pequeño de contraejemplos de ambas, obteniendo mediante el mismo de la intensidad entrópica de cada regla.

Una regla de inferencia (la Ley del Silogismo Hipotético) de la Lógica bivalente es la que fundamenta las demostraciones matemáticas y que afirma que $((\mathrm{p} \Rightarrow \mathrm{q} \wedge \mathrm{q}$ $\Rightarrow \mathrm{r}) \Rightarrow(\mathrm{p} \Rightarrow \mathrm{r})$ ), siendo $\mathrm{p}$, q y $\mathrm{r}$ proposiciones. Cuando se trabaja con cuasi-implicaciones de variables $v_{i}, v_{j}, v_{k}$, la implicación $\left(\left(v_{i} \Rightarrow v_{j} \Rightarrow \wedge v_{j} \Rightarrow v_{k}\right) \Rightarrow\left(v_{i} \Rightarrow v_{j}\right)\right)$, no es necesariamente una tautología y se cumple dicha ley únicamente si la intensidad de $\mathrm{v}_{\mathrm{j}} \Rightarrow$ $\mathrm{v}_{\mathrm{k}}$ es, al menos, de 0.5 [2].

Para obtener los resultados con ASI, se utiliza el software Clasification Hiérarchique Implicative et Cohesitive (CHIC) creado por Gras y sus colaboradores. Este software presenta mediante una tabla de doble entrada la intensidad de todas las implicaciones entre las variables, pero, a fin de destacar aquellas que resultan significativas incluye también un grafo ponderado, donde el peso de los arcos está dado por la intensidad de las implicaciones al cual Couturier [5] denomina "grafo implicativo". Generalmente se colorean con distintos colores los arcos: con rojo los de 0,99 , con azul las de 0,95 , con verde las de 0,90 y con gris las de 0,85 .

En este trabajo, para analizar las respuestas de todos los alumnos de Ingeniería se utilizó la implicación entrópica; en cambio, para analizar las de los estudiantes según la carrera a la que pertenecen, se usó la clásica. En ambas oportunidades (por ser todas las muestras aleatorias) las probabilidades se calcularon mediante la ley de Poisson.

Así pues, se trabajó con las respuestas de 207 alumnos: 110 de Ingeniería en Electrónica, 63 de Ingeniería Eléctrica y 44 de Ingeniería en Agrimensura.

\subsection{Uso del asi para el análisis del ejercicio 3 del test}

E1 ejercicio analizado consiste en determinar el valor de verdad de las siguientes 10 proposiciones, con las hipótesis de que a,b y c son números reales, mientras que $\mathrm{n}$ y $\mathrm{m}$ son naturales.

\section{Tabla 1: pág siguiente}

Se denota con $I_{j}$, con $1 \leq \mathrm{j} \leq 10$ a las 10 variables que se generan a partir del ejercicio propuesto. 


\begin{tabular}{|c|c|c|c|}
\hline $\mathrm{I}_{1}$ & $\frac{a b^{2}-1}{10 m-1}=\frac{a b^{2}}{10 m}$ & $\mathrm{I}_{6}$ & $(a \cdot b)^{3}=a^{3} \cdot b^{3}$ \\
\hline $\mathrm{I}_{2}$ & $\begin{aligned} \frac{3 a}{n}-\frac{10 b^{5}+3}{m} & \\
= & \frac{3 a m-10 n b^{5}-3 n}{n \cdot m}\end{aligned}$ & $\mathrm{I}_{7}$ & $\left(a^{n}\right)^{\mathrm{m}}=\mathrm{a}^{\mathrm{n} \cdot \mathrm{m}}$ \\
\hline $\mathrm{I}_{3}$ & $\frac{a c^{2}+2 b}{c^{2}}=a+\frac{2 b}{c^{2}}$ & $\mathrm{I}_{8}$ & $\begin{array}{l}a^{m} \cdot a^{n}= \\
\left(a^{3}\right)^{m+n+n}\end{array}$ \\
\hline $\mathrm{I}_{4}$ & $a^{2}-b^{2}=(a-b) \cdot(a+b)$ & $\mathrm{I}_{9}$ & $+\sqrt{a^{2}}=a$ \\
\hline$I_{5}$ & $a^{0}=1$ & $\mathrm{I}_{10}$ & $\frac{m}{\sqrt{m}}=\sqrt{m}$ \\
\hline
\end{tabular}

TABLA 1. Variables en estudio

\begin{tabular}{|c|c|c|c|c|c|c|c|c|}
\hline & \multicolumn{2}{|c|}{$\begin{array}{l}\text { Total } \\
\text { Ingenierías }\end{array}$} & \multicolumn{2}{|c|}{$\begin{array}{l}\text { Ingetioria en } \\
\text { Agrimensura }\end{array}$} & \multicolumn{2}{|c|}{$\begin{array}{l}\text { Ingenieria } \\
\text { Eléctrica }\end{array}$} & \multicolumn{2}{|c|}{$\begin{array}{l}\text { Ingetiteria } \\
\text { elı } \\
\text { Electrónica }\end{array}$} \\
\hline & $\bar{x}$ & $s$ & $\bar{x}$ & $s$ & $\bar{x}$ & $s$ & $\bar{x}$ & $s$ \\
\hline il & 0,43 & 0,49 & 0,52 & 0,50 & 0,50 & 0,50 & 0,36 & 0.48 \\
\hline 12 & 0,29 & 0,45 & 0,37 & 0,48 & 0,25 & 0.43 & 0,28 & 0,45 \\
\hline i3 & 0,34 & 0,47 & 0,35 & 0,48 & 0,41 & 0,49 & 0,32 & 0.47 \\
\hline I4 & 0,65 & 0,47 & 0,65 & 0,48 & 0,68 & 0.47 & 0,65 & 0,48 \\
\hline 15 & 0,07 & 0,26 & 0.05 & 0,21 & 0,09 & 0,29 & 0,09 & 0,29 \\
\hline I6 & 0,66 & 0,47 & 0,54 & 0,50 & 0,80 & 0,40 & 0,69 & 0.46 \\
\hline$I]$ & 0,78 & 0,40 & 0,81 & 0,39 & 0.73 & 0,45 & 0,80 & 0.40 \\
\hline I8 & 0,50 & 0.50 & 0,48 & 0,50 & 0.61 & 0.49 & 0,47 & 0.50 \\
\hline 19 & 0,09 & 0,29 & 0,19 & 0,39 & 0,09 & 0,29 & 0,05 & 0.21 \\
\hline I10 & 0,29 & 0.45 & 0,19 & 0,39 & 0.43 & 0,50 & 0,31 & 0.46 \\
\hline
\end{tabular}

Tabla 1. Estadisticos descriptivos seguin carreras 


\subsection{Resultados}

En principio se realizó un análisis estadístico descriptivo de las respuestas del total de los alumnos y, también, discriminados por carrera (Tabla 1).

Al observar la Tabla 1 se advierte que el $78 \%$ de los estudiantes reconoció la validez de la propiedad de potencia de otra potencia $\left(i_{7}\right)$ siendo éste el ítem que ostenta la mayor frecuencia de aciertos. En cambio, los errores más frecuentes tienen que ver con las definiciones de potenciación y de valor absoluto ( $i_{5} \mathrm{e}_{9}$, respectivamente)

Al analizar las respuestas por carrera, se observa que dentro de cada carrera estos resultados se mantienen. Sólo cabe destacar que el 80\% de los alumnos de Ingeniería Eléctrica responden correctamente $i_{6}$ (propiedad distributiva de la potenciación respecto a la multiplicación), superando el porcentaje de aciertos de los de las otras 2 carreras en más de 10 puntos.

Al aplicar el ASI se obtuvo el grafo implicativo de la Figura 1. Se observa que las reglas de mayor intensidad son: $\mathrm{I}_{9} \Rightarrow \mathrm{I}_{7}, \mathrm{I}_{9} \Rightarrow$ $\mathrm{I}_{5} \mathrm{e} \mathrm{I}_{7} \Rightarrow \mathrm{I}_{3}$ con una intensidad de 0,9 y con 0,99 de intensidad $I_{1} \Rightarrow I_{3}$ e $I_{3} \Rightarrow I_{6}$.

Dado que $\phi\left(\mathrm{I}_{7}, \mathrm{I}_{3}\right)=0,9$ y $\phi\left(\mathrm{I}_{3}, \mathrm{I}_{6}\right)=0,99$ es decir mayores que 0,5 , puede establecerse que $I_{9} \Rightarrow I_{3}$ e $I_{1} \Rightarrow I_{6}$

Interpretamos, a continuación, qué relaciones conceptuales implican estas 7 reglas:

- $\mathrm{I}_{1} \Rightarrow \mathrm{I}_{3}: \mathrm{Se}$ evidencia que todo alumno que reconoce que los cocientes de I1 son distintos, reconoce que el cero no es un número real invertible, lo cual se explica por el hecho de que detectar que la igualdad de dos cocientes equivale a la de los productos cruzados, supone una mayor complejidad cognitiva que la necesaria para detectar que el cero no es invertible, lo cual queda establecido en el axioma de existencia de inverso de todo número real no nulo.

- $\mathrm{I}_{3} \Rightarrow \mathrm{I}_{6}$ : Es frecuente que los alumnos tienen dificultad en identificar que en I3 el divisor puede ser nulo, mientras que en la proposición I6 que uno de los factores sea nulo no afecta su valor de verdad. De ahí que el sentido de la implicación indica que quienes logran establecer esta relación han dado un paso más que quienes no la establecen, en la evolución del pensamiento aritmético al algebraico.

- $\mathrm{I}_{9} \Rightarrow \mathrm{I}_{7}$ : Esta regla vincula la definición de valor absoluto con la propiedad de potencia de una potencia. Nótese que en la definición mencionada subyace una especie de "potencia de una potencia" que - obviamente - no es válida al ser uno de los exponentes un número racional no entero (1/2). Así pues, los estudiantes que establecen esta regla tienen en claro que dicha propiedad de la potenciación es válida sólo para exponentes enteros.

$\mathrm{I}_{9} \Rightarrow \mathrm{I}_{5}$ : Para determinar que $\mathrm{I}_{9}$ es falsa es necesario conocer las definiciones de valor absoluto, potenciación y radicación en $\mathrm{R}$, en cambio en el ítem 5 es suficiente conocer de manera completa la definición de potenciación. Estas diferencias explican el sentido de la regla.

$\mathrm{I}_{7} \Rightarrow \mathrm{I}_{3}$ : El sentido de la implicación esta dado porque las propiedades de 
la potenciación demandan un nivel de conocimiento superior al que requiere $\mathrm{I}_{3}$ que involucra sólo suma y producto y la segunda potencia de un número real, así como también la no existencia de inverso de cero en $\mathrm{R}$.

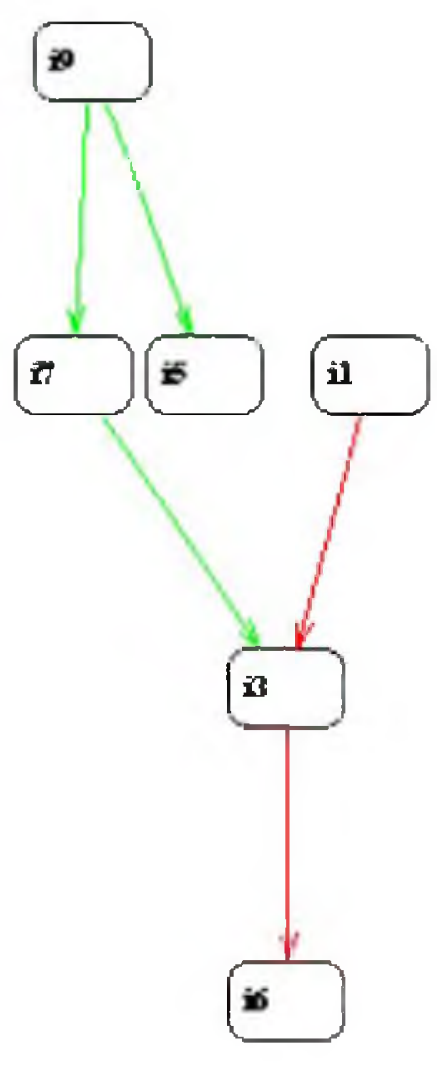

Figura 1. Grafo implicativo.

Alumnos de las carreras de Ingenieria de FACENA

$\mathrm{I}_{9} \Rightarrow \mathrm{I}_{3}$ : En esta regla se evidencia que el conocimiento de la definición de valor absoluto es más complejo que el saber que implica que el cero no es invertible. Quizás porque en el segundo la restricción esta dado sólo sobre el cero, mientras que $\mathrm{I}_{9}$ no se cumple para números negativos.

$I_{1} \Rightarrow I_{6}$ : Aquí es evidente que a los alumnos les resulta más sencillo identificar que el $I_{6}$ es verdadera, debido probablemente a que se refiere a una propiedad de potencia que proviene de un producto, que detectar la falsedad de $\mathrm{I}_{1}$ donde aparece un cociente.

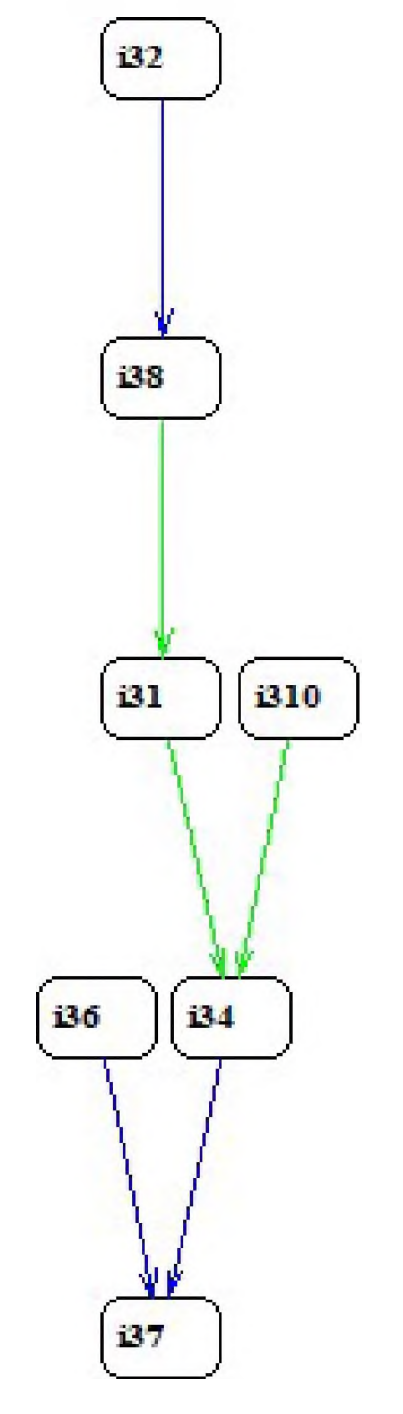

FIGURA 2. Grafo implicativo.

Ingeniería en Agrimensura

Al observar las Figuras 2 y 3, pueden detectarse las siguientes implicaciones comunes: 


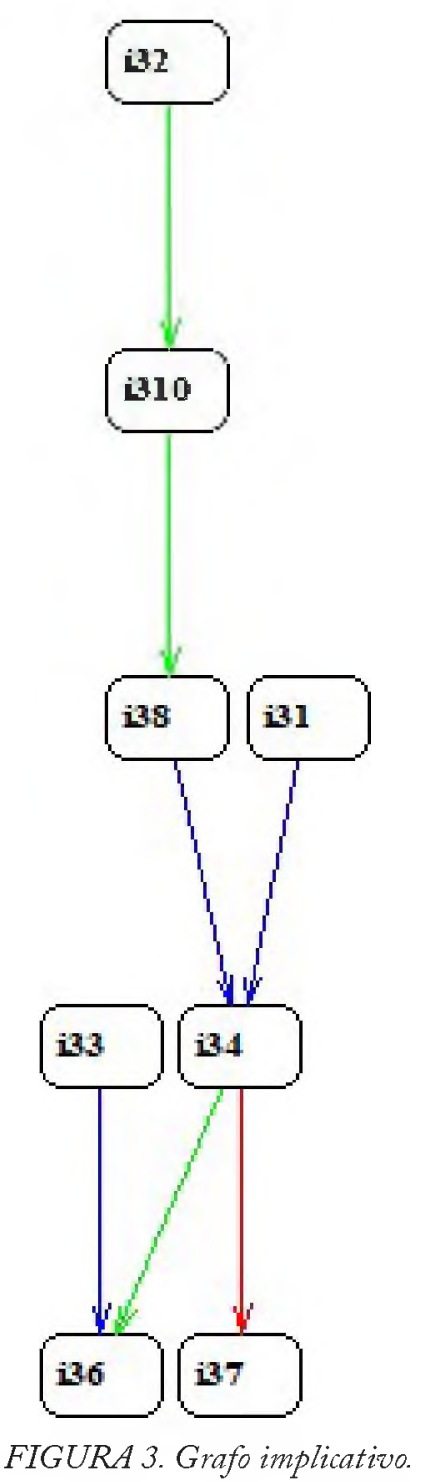

Ingeniería en Electrónica
- $\mathrm{I}_{8} \Rightarrow \mathrm{I}_{4}, \mathrm{I}_{10} \Rightarrow \mathrm{I}_{4}, \mathrm{I}_{2} \Rightarrow \mathrm{I}_{4}$ e $\mathrm{I}_{1} \Rightarrow \mathrm{I}_{4}:$ Observando estas implicaciones puede decirse que a los alumnos le resulta más sencillo reconocer una expresión equivalente a la diferencia de cuadrados que la de un producto de potencias de igual base, una expresión en la que se ha racionalizado su denominador o la igualdad entre dos cocientes.

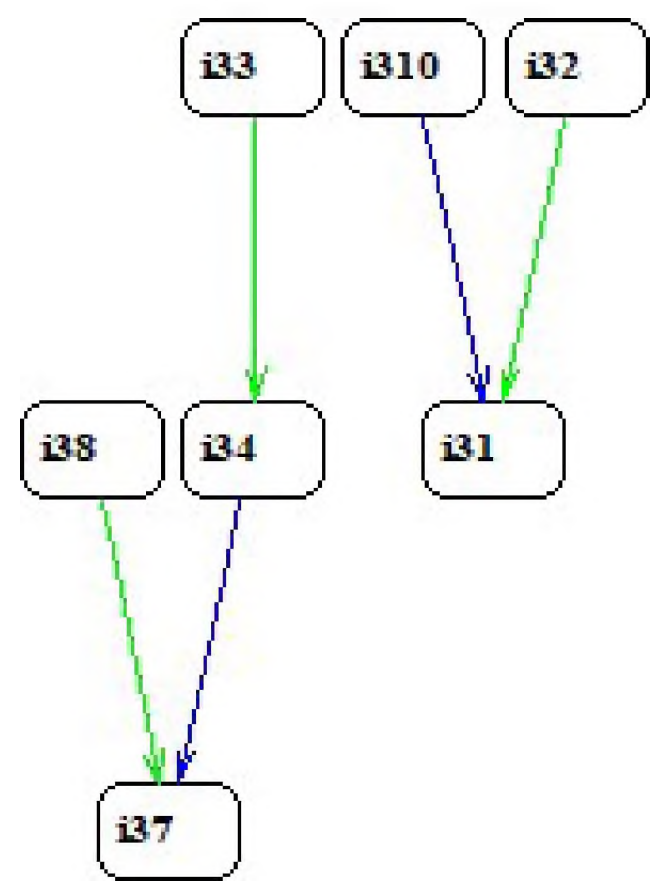

FIGURA 4. Grafo implicativo.

Ingenieria Eléctrica

- $\mathrm{I}_{4} \Rightarrow \mathrm{I}_{7}$ : Esta regla pone en evidencia que identificar una expresión equivalente a una diferencia de dos cuadrados, en la que se utiliza la propiedad distributiva del producto respecto a la suma y la cancelativa de la suma, implica un razonamiento más complejo que el necesario para la propiedad de potencia de potencia.

- $\mathrm{I}_{4} \Rightarrow \mathrm{I}_{7}, \mathrm{I}_{2} \Rightarrow \mathrm{I}_{7}, \mathrm{I}_{10} \Rightarrow \mathrm{I}_{7}$ e $\mathrm{I}_{1} \Rightarrow \mathrm{I}_{7}$ : Estas implicaciones resultan de la ley de silogismo hipotético, debido a que $\phi\left(\mathrm{I}_{4}, \mathrm{I}_{7}\right)>0,5$.

- $\mathrm{I}_{2} \Rightarrow \mathrm{I}_{\mathrm{o}}$ : Reconocer correctamente el valor de verdad de $\mathrm{I}^{2}$ significa que los alumnos conocen el algoritmo para la diferencia de dos cocientes, (el cual a su vez requiere el uso de la propiedad distributiva del producto con respecto a la suma), saber que es más 
complejo que la propiedad de producto de potencias de igual base.

$\mathrm{Al}$ observar las figuras 2 y 4 se detecta la regla $I_{2} \Rightarrow I_{1}$, donde se puede ver que a los alumnos les cuesta más identificar que los cocientes en el $\mathrm{I}_{2}$ son iguales pues suelen omitir la propiedad distributiva del producto respecto a la suma, al restar dichos cocientes.

Podemos rescatar que en las 3 carreras se presenta la implicación $I_{8} \Rightarrow I_{7}$, por tal motivo podemos concluir, que a los alumnos de las carreras de ingeniería les resulta más complejo utilizar la propiedad de producto de potencias de igual base que la propiedad de potencia de potencia.

\section{CONCLUSIONES}

De los resultados obtenidos, puede concluirse que hay muy pocos alumnos que han logrado realizar el pasaje de la Aritmética al Álgebra, el cual es uno de los objetivos prioritarios de la enseñanza de la Matemática en la Escuela Secundaria. Esta conclusión proviene del hecho de que la poca intensidad de las implicaciones halladas sugiere que dichas relaciones son débiles y establecidas por pocos estudiantes.

Estos resultados constituyen un importante aporte para orientar las actividades de enseñanza de los docentes de la cátedra en que se realizó el estudio en la búsqueda de actividades de enseñanza tendientes a superar las dificultades cognitivas señaladas.

\section{REFERENCIAS}

[1]Régnier J.C. (2013). Extracto de la obra Analyse statistique implicative. Une métho- de d'analyse de données pour la recherche de causalités". Gras, R.; Régnier J.C.; Guillet F. (Eds) (2009). Web:http://sites.univlyon2.fr/asi7/?page=0\&lang=es . Accedido:29/02/16.

[2] Gras, R.; Kuntz, P. (2009). El análisis estadístico implicativo (asi) en respuesta a problemas que le dieron origen". en teoría y aplicaciones del análisis estadístico implicativo. Primera aproximación en lengua hispana. Compiladores: Orús, P.; Zamora, L.; Gregori, P. (Eds). Stgo. de Cuba, Cuba: Universitat Jaume I de Castellón y Universidad de Oriente. pp $3-50$.

[3] Bodin, A. Analyse implicative: modèles sous-jacents à l'analyse implicative et outils complémentaires".(1995). Publications de l'Institute de Recherche Mathématiques de Rennes, fascicule 3: Fascicule de didactique de mathématiques ie.i.a.o." exp no 3, pp 1-23.

[4] Caputo, L.; Jorge, M.; Espinoza, R.; Porcel, E.; Romero, J. (2016). Análisis estadístico implicativo de los conocimientos previos sobre números reales de ingresantes a la universidad". Cadernos do IME - Série Estatística. Volumen 42, p 30 - 44. Río de Janeiro, Brasil: Universidade do Estado do Rio de Janeiro. ISSN impreso 1413 - 9022. ISSN on line $2317-4535$.

[5] Couturier, R. (2009). Chic: utilización y funcionalidades. en Teoría y aplicaciones del análisis estadístico implicativo. Primera aproximación en lengua hispana. Compiladores: Orús, P.; Zamora, L.; Gregori, P. (Eds). Stgo de Cuba, Cuba: Universitat Jaume I de Castellón y Universidad de Oriente. pp $51-64$. 